\title{
Menschenrechte, Institutionen und moralische Arbeitsteilung ${ }^{1}$
}

\author{
Bernd Ladwig
}

\section{Human Rights, Institutions, and the Division of Moral Labor}

Abstract: Are human rights general moral norms or are they conceptually tied to the political function of setting limits to the sovereignty of states? The essay steers a middlecourse in conceptualizing human rights as basic norms of political morality. Human rights emerge out of general moral claims held by individual human beings simply as such, but they play a particular role as necessary conditions of political legitimacy. Nevertheless, we should not identify them with those norms whose violation might justify restrictions on the sovereignty of other states. Such an equation would result in an all too minimalist conception of human rights. The essay defends a more ambitions conception. It integrates civil rights and human rights, particular states and global politics, democracy and collective self-determination under the perspective of a division of moral labor.

Keywords: Human rights, civil rights, political institutions, division of moral labor, international relations

Schlagwörter: Menschenrechte, Bürgerrechte, politische Institutionen, moralische Arbeitsteilung, Internationale Beziehungen

\section{Einleitung}

Allen Buchanan (2010, S. 680-681)² zufolge durchzieht eine Dichotomie die Philosophie der Menschenrechte. Denker wie James Griffin (2008) begriffen die Menschenrechte als allgemeine moralische Rechte. Autoren wie der späte Rawls (2002), Charles Beitz (2009) und Joseph Raz (2010) betonten dagegen, dass Menschenrechte vor allem bemüht werden, um staatliche Souveränitätsansprüche zu begrenzen. Für Vertreter der moralischen Lesart ist der normative Bezug auf interund transnationale Institutionen und Praktiken kein begriffliches Merkmal der Menschenrechte; er wird erst auf der Ebene der Rechtsanwendung relevant. Dagegen betrachtet Rawls die Menschenrechte von vornherein als Kernstück einer philosophischen Konzeption des Völkerrechts. Beitz möchte sie auf dem Wege einer kritischen Rekonstruktion der internationalen Praxis von Recht und Politik verständlich machen. Und Raz hebt hervor, dass die Menschenrechte einer vorpolitischen Fundierung weder fähig seien noch bedürften: Wir sollten nicht nach einer moralisch zwingenden Begründung für sie suchen, sondern anerkennen, dass sie von den Kontingenzen der internationalen Beziehungen abhängen.

1 Für hilfreiche Hinweise danke ich Andreas Oldenbourg sowie den beiden anonymen Gutachtern der PVS.

2 Vgl. auch die Beiträge in Besson u. Tasioulas 2010, Section VIII. 
Doch diese Dichotomie ist nicht erschöpfend. ${ }^{3}$ Das möchte ich zeigen, indem ich den Vorschlag entfalte, die Menschenrechte als Grundnormen ${ }^{4}$ der politischen Moral zu verstehen. Die Menschenrechte bedürfen durchaus einer moralischen Begründung; diese bildet die Bedingung ihrer Existenz. Doch die institutionalisierte und auf Institutionen bezogene Praxis der Politik bildet den Kontext menschenrechtlicher Geltung. In dieser zweiten Hinsicht haben die Vertreter politischer Menschenrechtsauffassungen recht: Menschenrechte sind nicht gleichzusetzen mit allen möglichen moralisch gültigen Ansprüchen von Menschen einfach als solchen. Im Unterschied zu anderen ,Rechten des Menschen` - wie ich die übergreifende Kategorie nennen werde - sind sie bereits begrifflich auf politische Institutionen und Praktiken bezogen.

Anders als Rawls, Beitz und Raz behaupten, muss dieser Kontext aber nicht unbedingt ein internationaler sein. Menschenrechte normieren internationale Beziehungen und innerstaatliche Verhältnisse, und letztere nicht nur insofern, als dabei die Souveränitätsansprüche der Staaten auf dem Spiel stehen. Wir sollten sie darum auch nicht mit Normen gleichsetzen, deren Verletzung wirtschaftliche Sanktionen oder militärische Eingriffe rechtfertigen könnte. Eine solche Gleichsetzung liefe auf ein zu minimales Verständnis menschenrechtlicher Inhalte hinaus. Ein anspruchsvolleres Verständnis kann man gewinnen, indem man Bürger- und Menschenrechte, Einzelstaaten und globale Politik, Demokratie und kollektive Selbstbestimmung unter dem Gesichtspunkt moralischer Arbeitsteilung miteinander vermittelt. Dies zu zeigen, ist der Zweck dieses Artikels.

\section{Menschenrechte als Grundnormen der politischen Moral ${ }^{5}$}

Menschenrechte, so meine Ausgangsannahme, sind moralische Normen, die uns in den öffentlichen Kontexten unserer Zuständigkeit in die Pflicht nehmen. „Moralisch" nenne ich Normen, die durch allgemein teilbare Gründe gerechtfertigt sind, so dass alle möglichen Normadressaten sie in ihr Gewissen aufnehmen können. Allgemein teilbar sind Gründe, die unabhängig davon gelten, an welcher speziellen Stelle im sozialen Kräftespiel jemand steht oder welche besonderen Vorlieben oder Abneigungen er hat (Ladwig 2011, S. 31). Auch die Menschenrechte bilden ein moralisch begründetes Gefüge von Normen. Doch sind sie nicht zuletzt dazu da, Machthaber im Hinblick auf soziale Grundordnungen an Zwecke der Machtunterworfenen zu binden. Ihre Beachtung ist eine notwendige Bedingung der Anerkennungswürdigkeit politischer Ordnungen, Programme und Prozesse.

Weil die Menschenrechte keine gewöhnlichen moralischen Rechte sind, sollten sie von anderen Rechten, die wir einfach als Menschen haben, unterschieden werden. Sicher ist etwa das Recht, nicht grundlos angelogen zu werden, ein gültiger

3 Mit dem folgenden Versuch, über sie hinauszugelangen, stehe ich nicht allein da. So haben jüngst Pablo Gilabert (2011) und Laura Valentini (2011) vorgeschlagen, die politische mit der moralischen Dimension der Menschenrechte zu vermitteln. Für diesen Hinweis danke ich Andreas Oldenbourg.

4 Damit sind hier einfach grundlegend bedeutsame Normen gemeint; die spezielle Bedeutung des Begriffs „Grundnorm“ in Hans Kelsens reiner Rechtslehre spielt keine Rolle.

5 Siehe zu diesem Abschnitt ausführlicher: Ladwig 2013. 
moralischer Anspruch jedes einzelnen Menschen. Gleichwohl wäre es ungewöhnlich, in jedem Fall einer ungerechtfertigten Lüge von einer Menschenrechtsverletzung zu reden. Typisch ist vielmehr, dass wir eine Menschenrechtsverletzung monieren, wo die Missachtung einer Pflicht von einer Regierung ausgeht oder jedenfalls in deren Verantwortungsbereich fällt. Dazu kann auch gehören, dass die Regierung zwar nicht selbst menschenrechtliche Grundgüter versehrt, deren Versehrung durch Dritte innerhalb ihres Machtbereichs aber nicht verhindert, obwohl sie dies könnte.

Wir sollten daher unterscheiden zwischen „Rechten des Menschen“ in einem allgemeinen und „Menschenrechten “ in einem besonderen Sinne. ${ }^{6}$ Unter den „Rechten des Menschen“ verstehe ich alle generellen moralisch gültigen Ansprüche, die wir ohne weitere Voraussetzung einfach als Menschen besitzen. Die grundlegenden Rechte dieser Art regeln die Interaktionen aller einzelnen Menschen untereinander. Wir schulden einander die Befolgung von Normen der Nichtschädigung und Nichtdemütigung, der Freiheitsbeachtung, der Fairness und Verlässlichkeit sowie der Hilfe in Notlagen. ${ }^{7}$ Dabei beziehen wir uns auf Güter, die für Menschen als soziale und kommunikative, vernunft- und moralfähige Leib- und Lebewesen (Höffe 2009, S. 240-241) grundlegend oder zentral bedeutsam sind. Auch die Menschenrechte fallen unter die Rechte des Menschen im ganz allgemeinen Sinne. Was sie von anderen Rechten dieser Art unterscheidet, ist der spezifische Kontext ihrer Geltung, mit dem auch eine Spezifizierung ihres Gehalts einhergeht.

Menschenrechte bilden Schutzvorrichtungen gegen Standardbedrohungen (Shue 1980, S. 13; 29-31), die uns mit unserer politischen Zuständigkeit, als Bürger oder auch als Amtsinhaber, konfrontieren. ${ }^{8}$ Standardbedrohungen bestehen in Gefahren, mit denen wir unter bestimmten Umständen rechnen müssen, weil sie in den Umständen systematisch angelegt sind. Menschenrechtlich erheblich sind solche Gefahren, sofern sie menschenrechtlich bedeutsame Güter betreffen. Ein klassischer Fall ist das Gewaltmonopol der Staaten. Diese verkörpern eine ständige Bedrohung für Leib, Leben und Grundfreiheiten der Menschen, die sich in ihrem Machtbereich befinden. Diese Art von Standardbedrohung hebt hervor, wer Menschenrechte jedenfalls vor allem als Schutzvorrichtungen gegen staatliche Gewalten versteht. Aber leidlich funktionierende Staaten spielen noch eine weitere menschenrechtliche Rolle: Sie sind auch dazu da, Schutz und Hilfe im Angesicht von Standardbedrohungen zu gewähren, die von anderen sozialen Mächten und Gewalten, wie den Verwertungszwängen der kapitalistischen Wirtschaft, ausgehen.

Diese Sichtweise entspricht der Gegenstandsbestimmung der politischen Moral von Rechten und Gerechtigkeit durch Philosophen wie John Rawls (1975) und

6 Die folgende Unterscheidung ist angeregt von Gewirth 1996, S. 9-10; sie findet sich dort allerdings nur der Sache nach, nicht auch explizit. Siehe dazu auch Pogge 2002, S. 65.

7 Hinzu kommen Normen der Fürsorglichkeit, die Beziehungen zwischen einseitig abhängigen Menschen und deren Bezugspersonen regeln.

8 Das müssen keine politisch erzeugten Bedrohungen sein; auch Naturkatastrophen etwa können unsere kollektive oder korporative Verantwortung als politische Akteure auf den Plan rufen. Das gleiche gilt für einige angeborene oder aus Krankheiten und Unfällen resultierende Beeinträchtigungen; für diesen letzten Hinweis danke ich einem der beiden anonymen Gutachter. 
Thomas Pogge (2002, S. 44-45). Rawls und Pogge plädieren für ein institutionalistisches Verständnis der politischen Moral: Deren wichtigster Gegenstand sei die gemeinsam verantwortete gesellschaftliche Grundordnung. Das schließt Urteile über das Tun und Unterlassen staatlicher Akteure ein, geht aber über diese hinaus. Auch institutionelle Komplexe wie die kapitalistische Markt- und Geldwirtschaft oder die Familien sind wesentlich dafür, wie eine soziale Ordnung Rechte und Pflichten sowie die Vorteile und Lasten der sozialen Zusammenarbeit verteilt. Zu sozialen Grundordnungen gehört zudem eine Ausdrucksdimension, die für manche menschenrechtlichen Normen, wie das Diskriminierungsverbot, besonders bedeutsam ist. ${ }^{9}$

Das institutionalistische Verständnis gibt uns ein - grobes - Kriterium, um zu erkennen, was die Menschenrechte von anderen Rechten des Menschen, die in allen möglichen interaktionalen Kontexten gelten, unterscheidet. Da aber alle Rechte des Menschen dieselbe moralische Wurzel haben, sollte man auch etwas darüber sagen, wo und warum sie sich verzweigen. Wie gelangen wir von allgemeinen Rechten des Menschen in den spezifisch menschenrechtlichen Geltungsraum hinein? Die Antwort ist zweigeteilt. Erstens brauchen wir Institutionen, weil sonst viele Rechtspflichten zeitlich, sachlich und sozial unterbestimmt blieben. Das ist der wichtigste Grund, aus dem Immanuel Kant ([1797] 1968) den Eintritt in eine Rechtsordnung für kategorisch geboten hielt - jedenfalls soweit Menschen die Möglichkeit haben, einander im Raum zu begegnen und in Gestalt widerstreitender Ansprüche zu ,lädieren‘.

Zweitens verlangen die Rechte des Menschen in einigen besonders wichtigen Hinsichten wie Leib, Leben und Grundfreiheiten nach einer starken, zwangsbewehrten Form der Geltung. Dabei ist der Gedanke leitend, dass Subjekte von Rechten über sanktionsbewehrte Möglichkeiten des Einforderns verfügen sollten und dass moralische Sanktionen wie Groll und Empörung oftmals nicht genügen, um Rechtsansprüche effektiv geltend zu machen. Also müssen wir dafür eintreten, einige schwache, nur durch moralische Sanktionsmittel gedeckte Rechte in starke, zwangsbewehrte Rechte zu verwandeln. Aus diesen beiden Gründen, der Pflichtenbestimmung und der Rechtsdurchsetzung, sind wir moralisch dazu verpflichtet, Staaten oder vergleichbare Gefüge politischer Institutionen zu bilden (Tugendhat 1993, S. 348).

Dieser Transformationsschritt ist selbst moralisch begründet; er ist mitnichten, wie Georg Lohmann (1998, S. 90-91) vermeint, nur eine Frage der Klugheit. Schließlich schuldet nicht jeder einzelne je sich selbst die Verwandlung schwacher in starke Rechte, sondern jeder schuldet dies allen jeweils anderen. Sofern einige Ansprüche erstens als Rechte von Menschen moralisch begründet sind und zweitens als schwache Rechte allein nicht zureichend zur Geltung gelangen können, ist auch ihre Verwandlung in starke Rechte moralisch geboten. Mit eben diesem Transformationsschritt betreten wir das Geltungsgebiet der eigentlichen Menschenrechte: den Raum politischer und positiv-rechtlicher Institutionen und Praktiken. Und in diesem Raum werden dann auch neue Arten von Rechtsansprüchen,

9 Dies ist ein zentraler Aspekt der Status Egalitarian Function der Menschenrechte (dazu Buchanan 2013, S. 28-30). 
etwa auf Schutz vor staatlicher Willkür, auf demokratische Mitwirkung oder auf Materialisierung des Bürgerstatus, denkbar und bedeutsam. ${ }^{10}$

Ist aber die Pflicht zur Institutionalisierung selbst schon eine menschenrechtliche Rechtspflicht oder entspringt sie aus Rechten des Menschen in einem allgemeineren Sinne? Nichts spricht meines Erachtens dagegen, sie unter beiden Gesichtspunkten zu sehen: Sie hat einen menschenrechtlichen Gehalt, und sie bindet uns einfach als Menschen. In Analogie zu Niklas Luhmanns Konzept „struktureller Kopplung “ (Luhmann 1997, S. 92-94) könnten wir daher sagen: Die Pflicht, zur menschenrechtlichen Institutionenbildung beizutragen, ist der Ort ,konzeptioneller Kopplung' zwischen Menschenrechten und anderen Rechten des Menschen.

Ein anderer Ausdruck für diesen Doppelcharakter ist „natürliche Pflicht“. Allen Buchanan sagt in diesem Sinne, jeder einzelne Mensch habe eine begrenzte moralische Pflicht, allen Menschen Zugang zu Institutionen zu geben, die ihre grundlegenden Rechte gewährleisten (Buchanan 2004, S. 85-97; gestützt auf Rawls 1975, S. 135-139). Begrenzt ist die Pflicht, da sie selbst nicht sonderlich klar bestimmt ist und unter dem starken Vorbehalt des Verbots steht, Individuen zu überfordern. ${ }^{11}$ Natürlich ist sie, da sie weder freiwillige Akte wie Versprechen noch bereits bestehende Institutionen voraussetzt. Insofern ist sie in bloßen Rechten des Menschen begründet. Aber auch hinsichtlich ihres Gehalts ist sie keine gewöhnliche menschenrechtliche Pflicht. Vielmehr besteht sie in der menschenrechtlichen Pflicht höherer Stufe, die institutionellen Bedingungen zu schaffen, damit direkt güterbezogene Pflichten erfüllt werden können (Ashford 2006). Wo diese Bedingungen fehlen, bleiben moralisch noch so gut begründete Ansprüche

10 Es wäre darum verkehrt, die Menschenrechte extensional mit solchen Ansprüchen gleichzusetzen, die wir bereits in einem vorstaatlichen Stadium gehabt haben könnten (Beitz 2003, S. 41). Allerdings besteht auch, contra Beitz, eine Kontinuitätsbeziehung zwischen Rechten des Menschen im Allgemeinen und Menschenrechten im Besonderen. Das eben soll mit der Metapher gesagt sein, dass alle Rechte des Menschen dieselbe moralische Wurzel besäßen. Mit dieser Metapher wird zugleich die Trennlinie kenntlich, die zwischen meiner Position und derjenigen von Allen Buchanan (2013) verläuft. Buchanan begreift die Menschenrechte, die uns in politischen Kontexten interessieren, als Normen des internationalen Rechts. Für diese sucht er nach einer pluralistischen Rechtfertigung. Er wendet sich damit gegen die Ansicht, wir müssten Menschenrechte unbedingt als moralische Rechte begründen. Zum Beispiel sei ein Menschenrecht auf Gesundheitsversorgung auch ohne ein korrespondierendes moralisches Recht begründbar: Es könnte die soziale Solidarität und den sozialen Gesamtnutzen fördern, das Ideal einer humanen und anständigen Gesellschaft ausdrücken oder dazu beitragen, dass Menschen ihre Pflichten des Wohltuns erfüllen können (Buchanan 2013, S. 28). Aber dieses Beispiel macht auch drei Probleme sichtbar. Erstens sind Ausdrücke wie „Solidarität“, „sozialer Gesamtnutzen“ und „humane und anständige Gesellschaft" sehr vage, und manche Versuche ihrer Konkretisierung nehmen selbst auf moralische Rechte Bezug. Deutet man diese Ausdrücke aber unabhängig von moralischen Rechten des Menschen, so besteht zweitens keine Sicherheit, dass sich die vermeinten Güter am besten in der Form positiver Menschenrechte gewährleisten lassen. Und auch wenn dies manchmal der Fall ist, sind drittens Spannungen zwischen Menschenrechten und Werten wie Solidarität oder Zielen wie einem größtmöglichen Gesamtnutzen möglich. Für einen solchen Fall aber gibt uns Buchanans pluralistische Strategie der Rechtfertigung keinen kritischen Maßstab an die Hand, der eine Auflösung gerade im menschenrechtlichen Sinne verlangen würde. Wir sollten daher um der kritischen Funktion der Menschenrechte willen daran festhalten, dass diese als moralische Rechte von Individuen begründbar sein müssen - was zusätzliche, aber eben im Zweifelsfall nachgeordnete Gründe anderer Art nicht ausschließt.

11 Vielleicht sollten wir deshalb eher von einer bloßen menschenrechtlich begründeten „Verpflichtung" als von einer regelrechten „Pflicht" reden. 
zeitlich, sachlich und sozial unterbestimmt und die Kooperationsprobleme ihrer Durchsetzung harren einer Lösung.

Eine wichtige Implikation für den Rechtsbegriff sei erwähnt. Rechte sind gültig begründete Ansprüche, denen aber nicht auf jeder Stufe und unter allen Umständen schon starke und wohlbestimmte Pflichten entsprechen müssen. ${ }^{12}$ Man kann Rechte in allgemeinen Begriffen bezeichnen, etwa als gültige Ansprüche auf Schutz und Hilfe in Notlagen, ohne damit schon vorwegnehmen zu müssen, wer genau wem genau was genau wann genau schuldet. Dies gibt uns in vielen Fällen die nötige Flexibilität, um die von gültigen Ansprüchen ausgehenden Forderungen und deren Adressaten kontextspezifisch zu bestimmen. Wir gewinnen so ein angemessen dynamisches Verständnis der auf Rechtsgüter bezogenen Zuständigkeiten und Zumutungen (so auch Gilabert 2011, S. 456-457). Und manchmal ist die erste Zumutung, die von gültigen Ansprüchen ausgeht, die Forderung nach kollektivem Handeln zum Aufbau von Institutionen.

In diesem Sinne mögen selbst sogenannte Manifest-Rechte (Feinberg 1980, S. 153) eine orientierende Funktion erfüllen. Manifest-Rechte sind gültig begründete Ansprüche, denen aber noch keine zureichend bestimmten und effektiv erzwingbaren Gegenstück-Pflichten entsprechen, weil die Adressaten noch nicht existieren oder zur Pflichterfüllung noch außerstande sind. Soweit wir jedoch die Möglichkeit haben, einen solchen Adressaten durch kollektives Handeln aufzubauen, und soweit wir zu kollektivem Handeln unter Bedingungen halbwegs fairer Verantwortungsteilung in der Lage sind, ${ }^{13}$ haben wir die - höherstufige - Verpflichtung, dafür zu sorgen, dass ein Recht kein bloßes Manifest-Recht bleibt. Die effektive Einklagbarkeit bei ganz bestimmten Pflichtenträgern ist daher keine Existenzbedingung für ein beliebiges Recht. Vielmehr sollten wir umgekehrt sagen, die erfolgreiche moralische Begründung eines Anspruchs spreche allererst dafür, diesen so effektiv wie möglich zur Geltung zu bringen.

12 Dagegen behauptet Susan James (2003), die Erzwingbarkeit (enforceability) sei eine notwendige Bedingung für die Existenz beliebiger Rechte. Onora O’Neill (1996) vertritt die etwas schwächere These, Rechte existierten nur zusammen mit zureichend bestimmten und ganz bestimmten Akteuren zugewiesenen Gegenstück-Pflichten. Ich halte aber sowohl James' als auch O’Neills Vorstellung, was Ansprüche zu ,echten Rechten' mache, für zu eng und zu wenig beweglich: Eine von beiden Autorinnen vergessene Funktion der Berufung auf Rechte besteht darin, Akteuren Gründe zu geben, nach Mitteln und Wegen der Rechtsverwirklichung zu suchen und durch kollektives Handeln für deren Voraussetzungen zu sorgen.

13 Die Formulierung „halbwegs fair“ ist eine Verlegenheitslösung. Ich möchte mich damit um eine schwierige Frage herumdrücken, die einen eigenen Aufsatz wert wäre: Bin ich als bemittelter Mensch dazu verpflichtet, mehr zu tun, als ich tun müsste, wenn auch alle anderen ihren fairen Anteil trügen, nur weil einige diesen Anteil nicht tragen? Eine solche Verpflichtung erscheint unfair. Aber das strikte Beharren auf dem eigenen fairen Anteil hätte zur Folge, dass Menschenrechte unerfüllt blieben, die erfüllt werden könnten, ohne dass irgendein Akteur etwas von vergleichbarem Gewicht opfern müsste. Ich halte daher die Beschränkung unserer Verpflichtungen auf einen strikt verstandenen fairen Anteil unter der ,nichtidealen` Bedingung unvollkommener Normbefolgung für falsch. Irgendeinen schwächeren Fairness-Constraint aber sollten wir womöglich gelten lassen. Daher die Formulierung „halbwegs fair“; vgl. zu dieser schwierigen Kontroverse u. a. Singer 1972; Murphy 2000; Cullity 2007; Mieth 2012. Für den Hinweis auf diese Problematik danke ich Andreas Oldenbourg. 


\section{Menschenrechte als Angelegenheiten von globalem Belang?}

Die Menschenrechte sind, wie alle anderen Rechte des Menschen auch, moralisch begründete Ansprüche: Sie gelten normativ gesehen noch dann, wenn ihre faktische Geltung auf sich warten oder zu wünschen übrig lässt. ${ }^{14}$ Auch auf ihre - vermutete - Missachtung reagieren wir schließlich spezifisch moralisch, etwa mit Empörung (zu spezifisch moralischen Reaktionsweisen: Strawson 1962). Gleichwohl haben die Vertreter politischer Konzeptionen in einem zentralen Punkt recht: Die Menschenrechte sind bereits begrifflich auf politische Institutionen und Praktiken bezogen. Sie nehmen uns in den öffentlichen Kontexten unserer $\mathrm{Zu}$ ständigkeit in die Pflicht (dazu Menke u. Pollmann 2007, S. 38-40). Wir ziehen sie heran, um die Anerkennungswürdigkeit politischer Ordnungen und der durch diese beschirmten sozialen Grundordnungen zu prüfen. Die Beachtung der Menschenrechte bildet eine notwendige, wenn auch nicht hinreichende Bedingung der normativen Legitimität politisch-sozialer Ordnungen. ${ }^{15}$

Dies gilt, grundsätzlich gesehen, nach innen wie nach außen: Staaten, die systematisch die Menschenrechte verletzen, verlieren ihre Anerkennungswürdigkeit gegenüber ihren eigenen Bürgern wie gegenüber allen anderen Staaten. Darum könnte man meinen, dass Vertreter der politischen Lesart wie Rawls, Beitz und Raz auch mit ihrer weitergehenden These richtig liegen: Die Menschenrechte seien von vornherein als Grundnormen der internationalen Beziehungen aufzufassen. Sie dienten dazu, die einzelstaatliche Souveränität von außen zu begrenzen. ${ }^{16}$ Raz meint, diese weitergehende These sei nötig, um Gehalt und Grenzen menschenrechtlicher Ansprüche im Einklang mit der tatsächlichen menschenrechtlichen Praxis zu bestimmen. Doch das wäre allenfalls überzeugend, wenn man die Menschenrechte klar von Bürgerrechten wie dem politischen Wahlrecht trennen könnte. ${ }^{17}$ Das Wahlrecht wird ja gewöhnlich nicht so verstanden, dass seine Missachtung souveränitätserhebliche Eingriffe in andere Staaten rechtfertigen könnte. Auch alle halbwegs anspruchsvollen sozio-ökonomischen Rechte und Diskrimi-

14 Dagegen behauptet Jürgen Habermas: „Der Begriff des Menschenrechts ist nicht moralischer Herkunft, sondern eine spezifische Ausprägung des modernen Begriffs subjektiver Rechte, also einer juristischen Begrifflichkeit. Menschenrechte sind von Haus aus juridischer Natur" (Habermas 1996, S. 222; kursiv im Original). Was immer aber über die Herkunft des Begriffs subjektiver Rechte zu sagen sein mag: Geltungslogisch können Menschenrechte, solange sie noch nicht positiviert sind, sondern allenfalls positiviert werden sollten, nicht schon juridische Rechte sein. Differenziert dazu Lohmann 1998; Menke u. Pollmann 2007, 1. Kapitel. Zur Geschichte des Konzepts subjektiver Rechte siehe Tuck 1977.

15 Ich vernachlässige hier eine dritte Dimension, die zu einem unverkürzten Verständnis der Menschenrechte gehört: die positiv-rechtliche. Diese ergibt sich meines Erachtens direkt aus dem besten Verständnis des politischen Grundzwecks der Menschenrechte: der effektiven Bindung öffentlicher Gewalten an die Zwecke der Gewaltunterworfenen (dazu Ladwig 2013, S. 58-60).

16 So formuliert Raz (2010, S. 328): „Following Rawls I will take human rights to be rights which set limits to the sovereignty of states in that their actual or anticipated violation is a (defeasible) reason for action against the violator in the international arena."

17 Rawls (2002, S. 96) versucht eben dies: „Einige glauben, daß die Menschenrechte im Großen und Ganzen eben diejenigen Rechte seien, welche die Bürger in einer vernünftigen konstitutionellen demokratischen Ordnung genießen [...]. Im Recht der Völker dagegen bringen Menschenrechte eine Klasse besonders dringlicher Rechte zum Ausdruck, zum Beispiel die Freiheit von Sklaverei und Leibeigenschaft, die Freiheit (aber nicht die gleiche Freiheit) des Gewissens und die Sicherheit ethnischer Gruppen vor Massenmord und Genozid.“ 
nierungsverbote müssten aus der Liste der eigentlichen Menschenrechte (buman rights proper) herausfallen - was bei Rawls konsequenterweise auch der Fall ist. ${ }^{18}$

Das institutionalistische Menschenrechtsverständnis, das ich vertrete, stützt eine solche Verengung auf die Funktion der Souveränitätseinschränkung nicht. Es setzt die moralischen Gründe, die dafür sprechen, Staaten ihre Anerkennungswürdigkeit abzusprechen, nicht mit den rechtfertigenden Gründen für souveränitätserhebliche Eingriffe gleich. Staaten könnten ihre Legitimität, ihr moralisches Recht zu regieren, durch bestimmte Regelungen und Praktiken verspielen, ohne dass dies anderen Staaten automatisch ein Recht zu Eingriffen gäbe. ${ }^{19}$ Mögliche Beispiele wären eben Missachtungen bestimmter Bürgerrechte, sozio-ökonomischer Rechte und Diskriminierungsverbote.

In meinem Verständnis sind die Einzelstaaten nicht zuletzt dazu da, manche Menschenrechte in bürgerrechtlicher Gestalt zu verwirklichen. Ebenso sollen sie sozio-ökonomische Rechte, auch oberhalb der Ebene bloßer Subsistenzmittel, gewährleisten und gegen Diskriminierungen etwa aus Gründen des Geschlechts, der sexuellen Orientierung, der Herkunft oder des Glaubens vorgehen - ungeachtet der Folgen für ihre Souveränität, falls sie dies nicht tun. Das scheint mir ganz der von Raz angemahnten Orientierung an der tatsächlichen menschenrechtlichen Praxis zu entsprechen. ${ }^{20}$ Auch spricht dafür ein historischer Umstand: Die Menschenrechte sind zuerst als Legitimationsgrundlagen partikularer politischer Projekte zur Geltung gelangt. Die Berufung auf sie sollte die Emanzipationsansprüche des Dritten Standes, dessen revolutionäre Erhebung zur Nation, mit einer universalistischen Rechtfertigung versehen (Blättler 2000; siehe auch die Beiträge in Menke u. Raimondi 2011). Die praktische Form der Verwirklichung des Menschenrechts war in allererster Linie das Bürgerrecht (das sowohl Abwehr- und Prozessrechte als auch Rechte auf politische Mitwirkung einschloss). ${ }^{21}$

Gleichwohl lag darin auch ein transzendierendes Moment: Prinzipiell sollten sich alle Menschen überall dazu aufgerufen fühlen, ihre Staaten im menschenrechtlichen Sinne umzugestalten. Die neue Legitimationsgrundlage politischer Herrschaft war mit einer Realisierung nur an einigen Orten der Erde normativ nicht vereinbar. In diesem Sinne hat bereits Kant die Menschenrechte als Angelegenheiten begriffen, die alle Artgenossen etwas angingen. So sagte er über die Französische Revolution, diese finde „in den Gemütern aller Zuschauer (die nicht in diesem Spiele mit verwickelt sind) eine Teilnehmung dem Wunsche nach, die

18 Da Beitz ein breiteres und differenzierteres Verständnis der relevanten internationalen Praxis hat, kann er einige der minimalistischen Schlüsse, zu denen Rawls gelangt, vermeiden.

19 Diese Unterscheidung trifft auch Raz (2010).

20 In den Menschenrechtspakten von 1966 etwa sind (Bürger-)Rechte auf politische Beteiligung, Diskriminierungsverbote und sozio-ökonomische Rechte vorgesehen.

21 Eine dritte, mit Bürgerrechten nicht gleichbedeutende Rechtskategorie bilden die Grundrechte. Diese sind positivierte Rechte, die nicht durch einfache parlamentarische Mehrheit geändert werden können (Alexy1994, S. 406). Zu den Grundrechten gehören nicht nur die Bürgerrechte, sondern auch sogenannte Jedermanns-Grundrechte. Auch kann man Grundrechte begründen, ohne dazu menschenrechtliche Argumente zu bemühen (etwa auf rein rechtspragmatische Weise). Mich interessieren hingegen nur solche Rechte, die durch genuin menschenrechtliche Gründe gedeckt sind. Und meine Behauptung lautet, dass dazu auch Bürgerrechte zählen, deren Verletzung nicht hinreicht, um (in Raz' Sinne von defeasible reasons) souveränitätserhebliche Eingriffe in den rechtsverletzenden Staat zu rechtfertigen. 
nahe an Enthusiasm grenzt, und deren Äußerung selbst mit Gefahr verbunden war, die also keine andre, als eine moralische Anlage im Menschengeschlecht zur Ursache haben kann" (Kant [1798] 1991, S. A 154).

Diese Begeisterung wäre grundlos gewesen, hätten die Revolutionäre - deren umwälzendes Tun als solches Kant natürlich nicht billigen konnte - nicht für (Rechts-)Prinzipien gestritten, die universale Anerkennung verdienten. Kant verurteilte die Französische Revolution als Praxis, begrüßte aber die sie beseelende Idee eines Rechts jedes Menschen, als Bürger die Gesetzgebung des eigenen Gemeinwesens mitzuprägen. Und er wagte eine hochherzige Vorhersage: Das Streben nach einer republikanischen Verfassung sei eine so bedeutsame Begebenheit, dass kein kommendes Geschlecht es mehr vergessen werde. „Denn jene Begebenheit ist zu groß, zu sehr mit dem Interesse der Menschheit verwebt, und, ihrem Einflusse nach, auf die Welt in allen ihren Teilen zu ausgebreitet, als daß sie nicht den Völkern, bei irgend einer Veranlassung günstiger Umstände, in Erinnerung gebracht und zu Wiederholung neuer Versuche dieser Art erweckt werden sollte“ (Kant [1798] 1991, S. A 150).

Kant bringt hier zum Ausdruck, in welchem Sinne die Menschenrechte immer schon Angelegenheiten von globalem Belang gewesen sind. Sie sind es, so viel ist für den strikten Interventionsgegner Kant klar, sicher nicht im Sinne der Ermächtigung anderer Staaten zu souveränitätserheblichen Eingriffen. Die zuletzt zitierte Stelle wirkt vielmehr wie eine Vorwegnahme von Michael Walzers Idee des wiederholenden Universalismus (Walzer 1996, S. 143-147). Eine einmal lokal verwirklichte Idee von universeller Strahlkraft wird Menschen an anderen Orten und zu anderen Zeiten dazu bewegen, sie mit eigenen Mitteln und auf eigene Weise wiederum lokal zu verwirklichen. Und jeder solche Versuch wird die uneigennützigen Sympathien auch aller sozial Fremden finden, die die Grundidee teilen. In diesem Sinne sind Menschenrechte nie nur innere Angelegenheiten einzelner Staaten oder Bürgerschaften. Auch als Bürgerrechte sind sie jedenfalls insofern eine Angelegenheit aller, als jeder Mensch ein Recht auf Bürgerrechte hat. Diese sollen Bürgern zukommen, weil sie Menschen sind, und Menschen, soweit sie Bürger sind. Die Menschenrechte fundieren die Rechte von Bürgern und transzendieren sie zugleich. ${ }^{22}$

Diese knappe historische Rekonstruktion spricht gegen eine scharfe konzeptionelle Scheidung von Menschen- und Bürgerrechten. Den Zusammenhang zwischen beiden stiftet ein logisch höherstufiges Recht: Auch Bürgerrechte zählen zu den Menschenrechten, weil wir einen menschenrechtlichen Anspruch auf Bürgerrechte haben. So können wir Rechte, die bis auf weiteres nur Staatsbürger oder vielleicht auch ,Denizens، auf einem bestimmten Staatsgebiet wahrnehmen dürfen, wie das aktive und passive Wahlrecht, gleichwohl als menschenrechtlich geboten erkennen. Und wir dürfen, ganz im Sinne der Geschichte der Menschenrechte, daran festhalten, dass diese nicht zuletzt auf dem Boden bestehender Staaten lokale Legitimität stiften sollen (so auch Forst 2010, S. 726-727). Eine wesentliche Funktion der Menschenrechte liegt weiterhin darin, uns Richtlinien und Regeln für die Gestaltung staatlich geeinter Grundordnungen zu geben.

22 Erhellend zu diesem Verhältnis von universalen Menschen- und partikularen Bürgerrechten auch Wellmer 1999, S. 135-137. 


\section{Moralische Arbeitsteilung}

Die Idee moralischer Arbeitsteilung kann uns helfen, das Verhältnis von Partikularität zu Universalität der Menschenrechte angemessen zu begreifen (Goodin 1988; Shue 1988). Sie besagt, dass wir auch kosmopolitische Normen wie die Menschenrechte nicht unbedingt ,reichsunmittelbar' befolgen müssen. Wir können ihnen auch abgestuft nachkommen, indem wir jeder Stufe bestimmte Funktionen bei ihrer Verwirklichung zuweisen. Und in menschenrechtlicher Hinsicht mögen verschiedene Gründe dafür sprechen, die einzelstaatliche Stufe besonders stark zu betonen.

Dabei stehen die Staaten aber nicht zunächst normativ selbstgenügsam nebeneinander, um dann erst auf dem Wege des Vertragsschlusses auch zwischenstaatliche oder staatenübergreifende Verpflichtungen zu übernehmen. Vielmehr betrachte ich sie von vornherein als tragende Teile eines Systems, das als Ganzes nicht zuletzt dazu da ist, Menschenrechte zu realisieren. Das Staatensystem sollte eine Form sein, in der wir unsere natürliche Pflicht erfüllen: Es sollte jedem einzelnen Menschen Zugang zu Institutionen geben, die seine grundlegenden Rechte gewährleisten. Ich vertrete also kein Konsensmodell partieller staatlicher Souveränitätseinschränkungen, sondern ein Modell geteilter Verantwortlichkeit, aus dem ein Grundzweck von Souveränität überhaupt erst hervorgeht. ${ }^{23}$ Repräsentiert wird das System durch eine internationale Organisation, die möglichst alle Staaten umfassen sollte, heute also am ehesten durch die Vereinten Nationen. Aber in das System lassen sich prinzipiell auch nichtstaatliche Bestandteile wie Nichtregierungsorganisationen oder Unternehmen ${ }^{24}$ einfügen.

Im Völkerrecht wie auch in der politischen Philosophie herrscht die Ansicht vor, dass die primären Träger menschenrechtlicher Pflichten stets die Staaten seien. ${ }^{25}$ Überhöht man dies zu einer begrifflichen Wahrheit, so handelt man sich das folgende Problem ein: „If states are the sole bearers of the primary duties to implement human rights, this would have the peculiar upshot that a universal moral right ceases to be a human right simply because the primary responsibility for its fulfillment has shifted to non-state-organizations" (Tasioulas 2009, S. 945). Wir sollten darum den Zusammenhang zwischen Menschenrechten und Staaten als grundsätzlich kontingent ansehen. Staaten sind heute die wichtigsten, oder jeden-

23 Ein Grundzweck, denn erstens sind noch andere Grundzwecke, etwa der Schutz kollektiver Lebensformen, denkbar, und zweitens müssten wir, um moralisch verantwortlich über Reichweite und Grenzen der Souveränität zu befinden, auch die Folgen für Frieden und internationale Stabilität in Betracht ziehen. Dabei sind auch die Kräfteverhältnisse im internationalen System zu bedenken: Wir dürfen ökonomisch und militärisch überlegene Staaten nach Möglichkeit nicht zu einem Missbrauch ihrer Macht einladen (so auch Raz 2010).

$24 \mathrm{Zu}$ Unternehmen als moralischen, auch menschenrechtlich verpflichtungsfähigen Akteuren siehe Neuhäuser 2011.

25 So etwa Buchanan: „I shall argue that the basic idea of the system of international legal human rights is to develop a regime of international law whose primary function is to provide universal standards for regulating the behavior of states towards those under their jurisdiction, for the sake of those individuals themselves, conceived as social beings" (Buchanan 2013, S. 27; kursiv im Original). 
falls die anerkanntesten, ${ }^{26}$ politischen Herrschaftsorganisationen und als solche auch die zentralen Akteure und Adressaten im Völkerrecht. Hätte die Geschichte aber andere Organisationen als die politisch wichtigsten ausgelesen, so würden sich menschenrechtliche Erwartungen, meiner politisch-moralischen Konzeption zufolge, vor allem auf diese konzentrieren.

Selbst heute richten sich menschenrechtliche Erwartungen nicht exklusiv an Staaten, sondern in zunehmendem Maße auch an internationale Organisationen wie den Internationalen Währungsfonds, an große transnationale Unternehmen und sogar an Nichtregierungsorganisationen. Zumindest erwarten wir von nichtstaatlichen, aber politisch einflussreichen Organisationen, dass sie menschenrechtliche Respektspflichten beachten (Lafont 2011). In besonderem Maße verwerflich finden wir aber weiterhin die Missachtung menschenrechtlicher Respektspflichten durch staatliche Akteure; und vor allem diese sehen wir in der Verantwortung, menschenrechtliche Schutz- und Hilfspflichten zu erfüllen. Für diese verbleibende Sonderrolle der Staaten scheinen mir drei Arten von Gründen einschlägig, die grob gesprochen den drei vorherrschenden Konzeptionen in der heutigen politischen Philosophie entsprechen.

Unter dem liberalen Gesichtspunkt bestmöglicher Menschenrechtsverwirklichung spielen die Staaten eine besondere instrumentelle Rolle im Gefüge moralischer Arbeitsteilung. Unter dem republikanischen Gesichtspunkt eines intrinsischen Werts öffentlicher Mitwirkung geben sie Raum für politische Projekte, mit denen sich Menschen als Angehörige besonderer Bürgerschaften identifizieren können. Unter dem Gesichtspunkt einer Konzeption deliberativer Demokratie bilden sie institutionelle Bezugspunkte für eine argumentative Auslegung und Anverwandlung universalistischer Normen. Auf den zweiten und den dritten Gesichtspunkt werde ich im fünften Abschnitt mit Blick auf die Grundnorm kollektiver Selbstbestimmung zurückkommen. Der für diesen Artikel leitende Gesichtspunkt allerdings ist der liberale. Und mit diesem tritt die instrumentelle Bedeutung der Staaten für eine effektive und legitime Menschenrechtsverwirklichung hervor.

Staaten sind heute für eine effektive Mitwirkung an der Menschenrechtsverwirklichung besonders geeignet, weil und sofern sie mehrere Monopole, vor allem das Gewalt- und das Steuermonopol, auf sich vereinen. Zumindest die halbwegs funktionsfähigen unter ihnen können dadurch einzigartige Mittel zur Verfolgung menschenrechtlicher Zwecke - und freilich auch zu deren Vereitelung - mobilisieren. Mit Blick auf die Legitimität ist der Organisationszweck von Staaten bedeutsam: Staaten sind primär dazu da, ein Gemeinwohl zu verwirklichen. Die öffentlichen Räume der Rechtfertigung, die sie damit aufspannen, nötigen sie dazu, ${ }^{27}$ Ansprüche auf allgemeine Anerkennungswürdigkeit zu erheben, an denen jedenfalls die demokratischen unter ihnen auch effektiv gemessen werden können. Solche Staaten erfüllen darum in besonderem Maße die Bedingung der Rechenschaftspflichtigkeit (accountability) (dazu Schmelzle 2012). Dies wiederum qualifiziert sie in besonderer Weise zur Mitwirkung am arbeitsteiligen Projekt der

26 Die völkerrechtliche Anerkennung der Staaten ist von deren Funktionsfähigkeit weithin unabhängig; dazu Ladwig u. Rudolf 2011.

27 Im Sinne des von Jon Elster (1998, S. 104) erkannten strukturellen Zwangs zur Heuchelei. 
Menschenrechtsverwirklichung. Andere ressourcenstarke Akteure wie Unternehmen verfolgen dagegen andere primäre Organisationszwecke wie wirtschaftliche Gewinne. Und die Möglichkeiten, nichtstaatliche und selbst internationale Organisationen aus demokratischen Öffentlichkeiten heraus zur Rechenschaft anzuhalten, sind sehr begrenzt (Kreide 2007; Lafont 2012).

Gewiss, dies alles kann sich ändern, und es ist schon heute mit Blick auf viele Staaten eine steile Idealisierung (Risse 2011). Weder haben alle heutigen Staaten die nötigen Fähigkeiten zur effektiven Menschenrechtsverwirklichung noch können die Rechtssubjekte sie alle effektiv zur Rechenschaft ziehen. Nur soweit die Einzelstaaten die besten Bedingungen der Rechtsverwirklichung bieten können, sind sie darum auch die ersten Adressaten menschenrechtlicher Ansprüche (so auch Gilabert 2011, S. 457-458). Sie sind es nur insofern, als einzelstaatliche Lösungen genügen, wenn jeder Staat seiner Aufgabe für den eigenen Machtbereich nachkommen kann und auch tatsächlich nachkommt. Ihm dies abzuverlangen, fällt letztendlich in die Verantwortung jedes einzelnen Bürgers, der in seinem Gemeinwesen politischen Einfluss nehmen kann. Steht die Eigenart eines menschenrechtlichen Problems einer einzelstaatlichen Lösung entgegen, so müssen die Staaten ihre Handlungen aufeinander abstimmen und eventuell auch weitere Akteure in die Verantwortung ziehen. Wo die primär Verantwortlichen ganz oder teilweise versagen oder gar zu menschenrechtlichen ,Schurken' mutieren, geht jedenfalls ein harter Kern der Pflichten auf internationale Organisationen und letztlich auf die Staatengemeinschaft als Ganze über (Ladwig u. Rudolf 2011).

Versagen auch diese sekundär verantwortlichen Instanzen, so sind prinzipiell alle zurechnungsfähigen Individuen gefragt. Diese können allerdings auf kollektive und vor allem korporative Akteure nichtstaatlicher Art zurückgreifen, um ihre Verpflichtungen als Menschen möglichst koordiniert und mit dem nötigen Ressourceneinsatz zu erfüllen. Dabei ist die Kapazität zu helfen aber nur ein Kriterium der Pflichtenallokation neben anderen. Als weitere Kriterien kommen etwa die normative oder auch nur kausale Verantwortung für menschenrechtliche Probleme, das Profitieren von menschenrechtlichen Missständen oder vorgängige Beziehungen der Verbundenheit und des Vertrauens in Betracht. ${ }^{28}$ Nicht zuletzt müssen wir wiederum darauf achten, dass die Rechtssubjekte die Verantwortungsträger kontrollieren und zur Rechenschaft ziehen können. Auch dies wird das Problem der Unterbestimmtheit menschenrechtlicher Verpflichtungen auf der allgemein-menschlichen Ebene sicher nicht auflösen. Aber oft können wir mit etwas gutem Willen gute Gründe dafür finden, bestimmten Akteuren eine stärkere Zuständigkeit zuzuweisen als anderen.

Der Idealfall des Systems menschenrechtlicher Arbeitsteilung wäre eine Welt, in der jedes einzelne Rechtssubjekt für jedes seiner Menschenrechte die Sicherheit besäße, dass wenigstens eine Agentur das Rechtsgut verlässlich, diskriminierungs-

28 Diese und ähnliche Kriterien zieht David Miller (2007, S. 99-104) heran, um die menschenrechtliche Hilfsverantwortung (remedial responsibility) fair und wirksam zu verteilen. Miller fragt allerdings nicht nach Möglichkeiten der Allokation von Pflichten und Verpflichtungen auf einer allgemein-menschlichen Ebene. Vielmehr möchte er wissen, wofür nationale Gemeinschaften zuständig sein könnten. 
frei und zu tragbaren Kosten ${ }^{29}$ gewährleistet. Die heute und in absehbarer $\mathrm{Zu}$ kunft einzig realistische Möglichkeit, dem Ideal auch nur nahezukommen, besteht, wie angedeutet, in einer Abstufung und Verschränkung von Pflichten und Verpflichtungen verschiedenartiger Akteure. ${ }^{30}$ Menschenrechtliche Obligationen begegnen uns dabei auf zwei verschiedenen Ebenen: auf einer direkt güterbezogenen Ebene als solche des Respekts, des Schutzes und der Hilfe (wenn möglich zur Selbsthilfe); auf einer logisch höherstufigen Ebene als Pflichten und Verpflichtungen zur Bildung von Institutionen, um Pflichten auf der ersten Stufe möglichst effektiv erfüllen zu können.

Die Idee moralischer Arbeitsteilung hat mehrere Vorteile. Sie macht deutlich, in welchem Sinne die Menschenrechte immer schon Angelegenheiten von inter- und transnationalem Belang sind. Aber sie überspringt dabei nicht die Stufe der Einzelstaaten. Auch nimmt sie diese nicht nur unter dem Gesichtspunkt der Zulässigkeit oder gar Gebotenheit externer Interventionen in den Blick. In der moralischen Arbeitsteilung spielen die Staaten weiterhin auch die Rolle einer bürgerrechtlichen Realisierung menschenrechtlich begründeter Ansprüche. Ebenso tragen sie die Verantwortung für soziale Gewährleistungen noch oberhalb der Ebene bloßer Subsistenzmittel. Verfehlungen in solchen Hinsichten verdienen interne wie externe Kritik unabhängig davon, ob sie die Schwelle zur Rechtfertigung souveränitätserheblicher Eingriffe überschreiten. ${ }^{31}$ Und selbst wenn dabei Regierungen auf Sanktionsmittel wirtschaftlicher oder gar militärischer Art verzichten sollten, so sollten sie doch wenigstens bürgerschaftlichen Protest ermutigen und dessen Protagonisten zu schützen suchen. Das wohl wichtigste Mittel, um menschenrechtliche Verbesserungen anzustoßen, wird ohnehin innerstaatlich wie grenzüberschreitend der Druck bleiben, den soziale Bewegungen auf Regierungen und etwa auch auf Unternehmen ausüben (siehe dazu die Beiträge in Risse et al. 2013).

Die erfolgreiche Rechtfertigung souveränitätserheblicher Eingriffe ist nur ein Grenzfall des Umstands, dass Menschenrechte von Haus aus alle Menschen weltweit betreffen. Und von diesem Grenzfall her fällt keineswegs Licht auf das Geltungsgebiet der Menschenrechte im Ganzen. Wäre es anders, so dürften wir unter

29 Eine Minimalbedingung ist hier, dass die Kosten des Zugangs zu irgendeinem menschenrechtlichen Gut nicht so hoch sein dürfen, dass sie irgendein Subjekt der Menschenrechte effektiv daran hindern, Zugang zu irgendeinem anderen Gut zu finden, auf das dieses selbe Subjekt einen menschenrechtlichen Anspruch hat. Das Kostenkriterium untersteht also einer Konsistenzbedingung mit Blick auf das Menschenrechtsgefüge im Ganzen.

30 Siehe das komplexe Bild, das James Nickel (2007, S. 38-40) von der Verteilung menschenrechtlicher Obligationen zeichnet.

31 Man könnte den Eindruck gewinnen, dass Raz (2010, S. 336) diesen Unterschied einebnet. So schreibt er, dass Staaten in genuin menschenrechtlichen Hinsichten „do not enjoy immunity from interference regarding these matters“. Aber „interference“ in der Form menschenrechtlicher Kritik ist etwas völlig anderes als „interference“ in der Form souveränitätserheblicher Eingriffe. Dass menschenrechtsverletzende Staaten daran interessiert sind, diesen Unterschied zu verwischen, damit sie sich jedwede „Einmischung in ihre inneren Angelegenheiten“ wirksam verbitten können, ist eine Sache. Raz aber muss sich entscheiden. Die menschenrechtliche Praxis, auf die er sich beruft, sieht sehr viel mehr Möglichkeiten menschenrechtlicher Kritik vor, als sie an Gründen für souveränitätserhebliche Eingriffe gelten lässt. Raz könnte nun unabhängige Gründe für ein restriktiveres Menschenrechtsverständnis vorbringen. Doch damit entfernte er sich von der Praxis, die er zu deuten vorgibt. 
„Menschenrechten“ nur mehr jene schlechthin basalen Normen verstehen, die zum zwingenden Völkerrecht zählen: Verbot von Sklaverei und Völkermord, von Verbrechen gegen die Menschlichkeit und dergleichen. Das wäre jedenfalls mit Blick auf den Sachumfang der allgemeinen Erklärung der Menschenrechte von 1948 und der beiden Menschenrechtspakte von 1966 radikal revisionär. ${ }^{32}$

\section{Menschenrechte und Toleranz unter Völkern}

Das Gleiche gilt in geringerem Maße noch für den menschenrechtlichen Minimalismus des späten Rawls (2002). Auch dieser orientierte sich einseitig an der internationalen Funktion der Menschenrechte, die einzelstaatliche Souveränität zu begrenzen und zwangsbewehrte Eingriffe in andere Gemeinwesen zu legitimieren. In der Folge fallen nicht nur die demokratischen Rechte, sondern auch viele Diskriminierungsverbote (dazu Buchanan 2010) aus der Liste der Menschenrechte heraus. Mit dem Rawlsschen Menschenrechtsminimalismus wäre sogar ein „achtbarhierarchisches" Gemeinwesen vereinbar, welches Frauen oder ,Ungläubigen“ grundsätzlich den Zugang zu hohen Staatsämtern verwehrte.

Das mag in der Praxis solange unproblematisch bleiben, wie die fragliche Regelung, etwa aufgrund einer „Gemeinwohlvorstellung der Gerechtigkeit“, von allen gewollt wird. Was aber, wenn einzelne oder ganze Gruppen beginnen, die Privilegien der Männer und der ,Rechtgläubigen' anzufechten? Müssten dann alle externen Betrachter in menschenrechtlicher Hinsicht neutral bleiben? Und dürfte die Regierung, solange sie dabei nicht maßlos vorgeht, solche Bestrebungen unterdrücken, ohne sich menschenrechtliche Kritik gefallen lassen zu müssen? Sind nicht gerade Konflikte mit Machthabern und Privilegierten Nagelproben für den Stellenwert der Menschenrechte in einer Gesellschaft (so dezidiert Forst 2010, S. 728)?

Nun ist ein Grund, der Rawls zu seinem Minimalismus motiviert hat, die Sorge, dass eine längere Liste von Menschenrechten zu wenig Raum ließe für Toleranz unter den Völkern. Diese müssen ihre Staaten auch nach eigenen Vorstellungen gestalten können. An dieser Überlegung sind zwei Aspekte zu unterscheiden, ein fragwürdiger (a) und ein grundsätzlich gültiger (b). Der gültige Aspekt verweist auf den Stellenwert kollektiver Selbstbestimmung im System menschenrechtlicher Arbeitsteilung. Damit kommen zugleich der republikanische und der deliberative Gesichtspunkt der Würdigung von Staatlichkeit zu ihrem Recht, die bislang unterbelichtet geblieben sind. Ich werde diesen Aufsatz daher mit wenigen Bemerkungen zur kollektiven Selbstbestimmung beschließen, die die gebotene Toleranz unter den Völkern abstecken und verständlich machen sollen. Zunächst aber zum ersten, fragwürdigen Gesichtspunkt.

$32 \mathrm{Zu}$ einem solchen Ultraminimalismus neigt gleichwohl Ignatieff (2001). Von einem (Ultra-)Minimalismus menschenrechtlicher Inhalte sollte dagegen das Bemühen um eine möglichst minimale menschenrechtliche Rechtfertigung unterschieden werden (zu dieser Differenzierung Cohen 2004). Eine solche Rechtfertigung sollte kein unnötiges metaphysisches Gepäck mit sich führen, weil dies die Gefahr vernünftiger Nichtübereinstimmung erhöht; eine Rechtfertigung für Menschenrechte muss schließlich allen mündigen Menschen in allen Gesellschaften einleuchten können. Aber damit ist nicht gesagt, dass einzig die Gründe, die für Normen des zwingenden Völkerrechts sprechen, auch menschenrechtliche zwingende Gründe sein könnten. 
(a) Kritikwürdig ist die Vorstellung, dass wir auch als Liberale prinzipielle und nicht nur pragmatische Gründe dafür haben könnten, dezidiert nichtliberale Gemeinwesen und Gerechtigkeitsvorstellungen zu tolerieren. ${ }^{33}$ Dagegen spricht, dass die Gründe für Toleranz, die Liberale akzeptieren sollten, eben liberale Gründe sein müssen. Eine spezifisch liberale Begründung für Toleranz ist aber normativindividualistisch und gleichheitsbezogen (Kymlicka 1996): Sie erkennt jedem einzelnen Menschen ein Grund-Recht auf gleiche Achtung und Berücksichtigung durch die zwangsbewehrten Gewalten des Gemeinwesens zu, dem er angehört (Dworkin 1990, S. 298). Aus diesem Grund-Recht folgt, dass der Staat jedenfalls keine Vorstellungen und Praktiken unterdrücken darf, über die seine Bürger vernünftigerweise geteilter Meinung sein können. Und er darf Bürger in solchen Hinsichten nicht diskriminierend behandeln, weil er sonst Angehörige zweiter Klasse erzeugte.

Eben dies aber kennzeichnet die Verfassungen „achtbar-hierarchischer Völker“: Diese sind hierarchisch, weil sie verschiedene Klassen von Gesellschaftsangehörigen kennen. Rawls sagt, wir sollten solche Völker gleichwohl tolerieren, weil sie die Menschenrechte achten. Da jedoch die Verfassungen solcher Völker substanziell diskriminierend sind, achten sie durchaus nicht alle Menschenrechte, wie sie in der liberalen Toleranzvorstellung vorausgesetzt sind. Auch achten sie sie nicht um der Individuen selbst willen. Diese sind im öffentlichen Leben achtbar hierarchischer Völker allein als Mitglieder von Gruppen vorgesehen. Und nur als „solche Mitglieder haben Personen dann Rechte und Freiheiten, die es ihnen ermöglichen, ihre Pflichten und Verpflichtungen zu erfüllen und sich in einem achtbaren System der sozialen Kooperation zu engagieren“ (Rawls 2002, S. 83). Diese Begründung steht aber in Spannung zur normativen Funktion der Menschenrechte, Individuen auch gegen kollektive Zumutungen zu schützen und zu fördern. Steht die Integrität von Gruppen auf dem Spiel, so müsste Rawls konsequenterweise einräumen, dass diese den Vorzug verdient vor noch so dringenden Ansprüchen von Individuen.

(b) Grundsätzlich gültig ist dagegen das Motiv, dass die Menschenrechte auch einen Platz für kollektive Selbstbestimmung vorsehen sollten. An ihr teilnehmen zu können ist selbst ein Menschenrecht. Dieses schützt ein aus Sicht vieler Bürger besonders wichtiges, auch intrinsisch wertvolles Gut. Vor allem republikanische Theoretiker betonen, dass Staaten Schutz verdienen um der politischen Projekte willen, mit denen sich Menschen als Angehörige besonderer Bürgerschaften identifizieren können. Man kann Menschen demütigen, indem man ihnen in der bür-

33 Rawls drückt dies so aus: „Die Tatsache, dass wir eine liberale Gerechtigkeitskonzeption auf die Gesellschaft der Völker ausweiten, die erheblich mehr religiöse und andere umfassende Lehren einschließt als jede einzelne Gesellschaft, führt unausweichlich zur Toleranz, wenn die beteiligten Völker ihre Angelegenheiten untereinander durch öffentlichen Vernunftgebrauch regeln" (Rawls 2002, S. 21). Und er schließt daraus: „Wenn von allen Gesellschaften gefordert würde, liberale Gesellschaften zu sein, fehlte es der Idee des politischen Liberalismus an der gebührenden Toleranz gegenüber anderen Möglichkeiten gesellschaftlicher Ordnung (wenn es sie denn, wie ich annehme, gibt)“ (Rawls 2002, S. 71). Dafür, dass dies prinzipiell und nicht nur pragmatisch gemeint ist, spricht jedenfalls der Ausdruck ,gebührend“. 
gerschaftlichen Hinsicht ihres Selbstverständnisses keine Verantwortung zutraut (dazu grundlegend Margalit 1997).

Von kollektiver Selbstbestimmung in einem strengen und nichtideologischen Sinne kann allerdings nur die Rede sein, wenn alle mündigen Individuen, die zum ,Selbst' gehören, an dessen Gesetzgebung auch gleichberechtigt mitwirken können. Andernfalls würde das ,Selbst' ${ }^{\star}$, das definitionsgemäß alle umfasst, von einigen einseitig festgelegt. Das wäre, jedenfalls soweit dabei Bereiche vernünftiger Nichtübereinstimmung berührt werden, mit der Gleichheit aller Staatsangehörigen nicht vereinbar. Subjekte von Menschenrechten müssen das System der Rechte, das für sie gelten soll, auch autonom nachvollziehen und mitgestalten können; dieses reflexive Erfordernis der Menschenrechtsverwirklichung heben Theoretiker der deliberativen Demokratie hervor.

Dagegen glaubt Joshua Cohen (2004; ähnlich Altman u. Wellman 2009, Chapter 2), dass kollektive Selbstbestimmung auch ohne gleiche politische Rechte möglich sei. Erforderlich sei allein, dass die Inklusionsbedingung effektiver Mitgliedschaft für alle erfüllt ist. Eben diese Bedingungen zu sichern sei die Funktion der Menschenrechte. Sie sollten gewährleisten, dass die Interessen und Sichtweisen aller Gesellschaftsangehörigen substanziell wie prozedural, in Verfahren kollektiver Willensbildung, Beachtung finden. Dass die Willensbildung gleichwohl keine demokratische sein müsse, begründet Cohen damit, dass sich staatliche Gemeinwesen im Zuge ihrer Selbstbestimmung auch gegen ,liberale' Normen wie gleiche politische Rechte entscheiden könnten. Auch hebt er die Möglichkeit vernünftiger Nichtübereinstimmung hervor, die uns zur Toleranz anhalte. Aber wenn mein Argument richtig ist, so spricht diese letzte Möglichkeit für und nicht gegen gleiche politische Rechte: Es wäre unfair, wenn einige für alle Entscheidungen träfen, die Bereiche vernünftiger Nichtübereinstimmung berührten, ohne dass alle an den Entscheidungen als Gleiche mitwirken könnten.

Auch wirkt es paradox, von einer kollektiven Entscheidung gegen gleiche politische Rechte zu sprechen. Eine Entscheidung, die nicht auch möglich gewesen wäre, wenn alle effektiv gleiche Teilnahmechancen gehabt hätten, verdient nicht als wahrhaft kollektiv zu gelten. Und wie will man erkennen, ob eine Entscheidung auch möglich gewesen wäre, wenn alle als Gleiche an ihr hätten mitwirken können? Offenbar, indem alle dies auch tatsächlich können, und nicht nur einmal, sondern immer wieder. Kurz, ohne demokratische Rechte keine wahrhaft kollektive Selbstbestimmung. Diese kann sich darum nicht gegen die Demokratie als solche wenden, ohne sich selbst zu verneinen. ${ }^{34}$

Aber die Menschenrechte sind umgekehrt auf kollektive Selbstbestimmung auch angewiesen. Und dabei haben die einzelnen staatlich geeinten Gemeinschaften Entscheidungsspielräume, die alle Gemeinwesen zur gegenseitigen Toleranz anhalten. Diese Forderung steht nur scheinbar im Gegensatz zum universalistischen Geltungsanspruch der Menschenrechte. Schließlich bedürfen die Menschen-

34 Davon zu unterscheiden ist allerdings die Wendung gegen ganz bestimmte, etwa konkurrenz- oder konkordanzdemokratische, parlamentarische, präsidentielle oder auch direktdemokratisch-partizipatorische Deutungen der demokratischen Grundnorm gleicher politischer Rechte und Wahlmöglichkeiten für alle. 
rechte als abstrakte Grundsätze der Konkretisierung. Sie sollen ja in allen möglichen kulturell geprägten Rechtsgemeinschaften zum Tragen kommen. Judith Butler verdeutlicht, dass dabei das Problem nicht in universalistischen Geltungsansprüchen als solchen, „sondern vielmehr in Anwendungen des Universalitätsgrundsatzes liegt, die kulturelle Besonderheiten außer Acht lassen und keiner Umformulierung in Reaktion auf ebenjene gesellschaftlichen und kulturellen Bedingungen zugänglich sind, die der Universalitätsgrundsatz in seinem Anwendungsbereich mit einschließt" (Butler 2007, S. 13).

Mit anderen Worten: Anwendungen universalistischer Grundsätze bedeuten immer auch eine Anverwandlung an die besonderen Merkmale des Kollektivs, dessen Angelegenheiten sie regeln sollen. Das heißt zum einen, dass dieselben Grundnormen nach verschiedenen Regelungen verlangen, je nachdem unter welchen faktischen Voraussetzungen etwa ökonomischer oder klimatischer Art sie zur Geltung gelangen sollen. Zum zweiten ist jeder Demos von seiner Geschichte geprägt; er steht unter dem Eindruck signifikanter Erfahrungen, die einen Demos vom anderen unterscheiden. Mit Blick auf die Menschenrechte sind dies vor allem Erfahrungen selbsterlittenen oder auch selbstverübten Unrechts. Auch die Menschenrechte erhalten darum im Streit um ihre angemessene Auslegung und Implementierung ein jeweils lokales Gepräge.

Aus diesem Grund ist ein „wiederholender Universalismus“ einem „Universalismus des allumfassenden Gesetzes“ vorzuziehen (Walzer 1996, S. 140-147). Die Divergenz der geschichtlichen Wege führt zu je eigenen Deutungen des Gehalts menschenrechtlicher Normen. So interpretieren Deutsche und US-Amerikaner die Religions- und die Meinungsfreiheit recht verschieden, und Deutsche sind für gruppendifferenzierte Rechte offener als Franzosen. Gewiss, längst nicht alle Bürger der jeweiligen Länder denken so und heißen ihre heimischen Regelungen gut; doch ihre politischen Kulturen geben die Auffassungsunterschiede deutlich genug zu erkennen.

Menschen neigen dazu, von ihrem geschichtlichen und geografischen Standort aus die Praktiken und Institutionen zu bevorzugen, die ihnen vertraut sind. Man könnte daher meinen, über die bestmögliche Auslegung menschenrechtlicher Grundsätze sei zwischen den Bürgern verschiedener Staaten gar kein fruchtbarer Streit möglich. Das erschiene mir vorschnell. Gerade weil wir zum Provinzialismus neigen, sollten wir für die Sichtweisen Fernstehender empfänglich sein (Sen 2010, S. 157; 178-179). Aber wiederum sollten wir auch mit der Möglichkeit vernünftiger Nichtübereinstimmung rechnen. Diese Möglichkeit besteht bereits unter den Bürgern eines und desselben Staates. Sie wird durch das internationale System jedoch potenziert, weil dieses zu einer Fragmentierung der Sichtweisen und maßgeblichen Erfahrungen führt.

Ob vernünftige Nichtübereinstimmung besteht, können wir immer nur ex post und bis auf weiteres wissen. Es ist allenfalls entscheidbar, nachdem fürs erste alle Argumente ausgetauscht worden sind. Wir sollten darum nicht aufhören, unsere Überzeugungen für wahr zu halten. Aber zugleich sollten wir zur Toleranz bereit sein und den Angehörigen anderer Staaten Spielräume für eigene Entscheidungen zubilligen. So bezeugen wir ihnen die gebotene Achtung für ihre von eigenen Erfahrungen geprägten Versuche der Menschenrechtsverwirklichung. Diese gehen 
zwar im letzten Grunde alle an, weil Menschenrechte als Grundnormen der politischen Moral immer schon Angelegenheiten von globalem Belang sind. Aber im arbeitsteiligen System ihrer Verwirklichung ist auch Raum für geschichtlich begründete und kulturell sedimentierte Differenzen. Diese dürfen nur nicht auf Kosten des moralischen Grundzwecks gehen. Er besteht darin, jeden einzelnen Menschen durch Gewährleistung grundlegender und zentraler Güter als ein Wesen eigenen Rechts zur Geltung zu bringen.

\section{Fazit}

Ich habe dafür plädiert, die Menschenrechte als Grundnormen der politischen Moral zu begreifen. Ihr bestimmender Zweck ist die effektive Bindung politischer Gewalten an die Zwecke der Gewaltunterworfenen. Von Menschenrechtsverletzungen reden wir mit Blick auf das Tun und Unterlassen politischer Autoritäten und auf die von diesen verantworteten gesellschaftlichen Grundordnungen. Der institutionalistische Zuschnitt der Menschenrechte unterscheidet diese von anderen Rechten des Menschen. Aber es sind die allgemeinen Rechte des Menschen selbst, soweit sie als starke Rechte gelten sollten, die uns den Schritt in den spezifisch menschenrechtlichen Geltungsraum moralisch vorschreiben.

Was die heute stark betonte internationale Dimension der Menschenrechte angeht, so spricht die politisch-moralische Konzeption für eine differenzierte Betrachtung. Auch ihr zufolge betreffen die Menschenrechte aufgrund ihres universalistischen Geltungsanspruchs und ihres normativen Gewichts als notwendige Bedingungen politischer Legitimität von vornherein alle Menschen weltweit. Gleichwohl haben sie nicht nur historisch vor allem staatlich begrenzte Ordnungen auf eine neue Legitimationsgrundlage gestellt. Noch heute ist der Einzelstaat ein besonders wichtiger Raum menschenrechtlicher Verwirklichung. Das heißt allerdings auch, dass seine Anerkennungswürdigkeit wesentlich davon abhängt, ob er seiner Verantwortung in einem System moralischer Arbeitsteilung gerecht wird. $\mathrm{Zu}$ diesem Zweck ist er schon heute auf die Kooperation auch mit Akteuren anderer Art angewiesen.

Das Recht auf souveränitätserhebliche Eingriffe in die Belange anderer Staaten ist eine extreme Konsequenz der globalen Geltung der Menschenrechte. Aber dies rechtfertigt keinen inhaltlichen Ultraminimalismus, der nur mehr Normen des zwingenden Völkerrechts als genuine Menschenrechte gelten ließe. Ebenso wenig spricht das vom späten Rawls betonte Toleranzgebot dafür, unsere menschenrechtlichen Ansprüche etwa um Diskriminierungsverbote oder demokratische Rechte zu verkürzen. Wofür es spricht, ist die kontextsensible Verwirklichung der Menschenrechte im Rahmen kollektiver Selbstbestimmung. Politische Gemeinschaften brauchen Spielräume für demokratische Entscheidungen, um die Menschenrechte auf ihre besonderen Voraussetzungen und Erfahrungen zuschneiden zu können. 


\section{Literatur}

Alexy, Robert. 1994. Theorie der Grundrechte. Frankfurt a. M.: Suhrkamp Verlag.

Altman Andrew, und Christopher Heath Wellman. 2009. A Liberal Theory of International Justice. Oxford: Oxford University Press.

Ashford, Elizabeth. 2006. The Inadequacy of Our Traditional Conception of the Duties Imposed by Human Rights. Canadian Journal of Law and Jurisprudence 19: 217-235.

Beitz, Charles. 2003. What Human Rights Mean. Daedalus 132: 36-46.

Beitz, Charles. 2009. The Idea of Human Rights. Oxford: Oxford University Press.

Besson, Samath, und John Tasioulas. (Hrsg.). 2010. The Philosophy of International Law. Oxford: Oxford University Press.

Blättler, Sidonia. 2000. Zwischen Universalismus und Nationalstaatskritik. Zum ambivalenten Status des Nationalstaats bei Hannah Arendt. Deutsche Zeitschrift für Philosophie 48: 691-706.

Buchanan, Allen. 2004. Justice, Legitimacy, and Self-Determination. Moral Foundations for International Law. Oxford: Oxford University Press.

Buchanan, Allen. 2010. The Egalitarianism of Human Rights. Ethics 120: 679-710.

Buchanan, Allen. 2013. The Heart of Human Rights. Oxford: Oxford University Press.

Butler, Judith. 2007. Kritik der ethischen Gewalt. Frankfurt a. M.: Suhrkamp Verlag.

Cohen, Joshua. 2004. Minimalism About Human Rights: The Most We Can Hope For? The Journal of Political Philosophy 12: 190-213.

Cullity, Garrett. 2007. Fremde in Not. Wohltätigkeit, Rechte und Staatsbürgerschaft. In Weltarmut und Ethik. Hrsg. Barbara Bleisch und Peter Schaber, 53-75. Paderborn: Mentis Verlag.

Dworkin, Ronald. 1990. Bürgerrechte ernstgenommen. Frankfurt a. M.: Suhrkamp Verlag. Elster, Jon. 1998. Deliberation and Constitution Making. In ders.: Deliberative Democracy, 97-122. Cambridge: Cambridge University Press.

Feinberg, Joel. 1980. The Nature and Value of Rights. In ders.: Rights, Justice, and the Bounds of Liberty: Essays in Social Philosophy, 143-158. Princeton, N. J.: Princeton University Press.

Forst, Rainer. 2010. The Justification of Human Rights and the Basic Right to Justification: A Reflexive Approach. Ethics 120: 711-740.

Gewirth, Alan. 1996. The Community of Rights. Chicago: The University of Chicago Press.

Gilabert, Pablo 2011. Humanist and Political Perspectives on Human Rights. Political Theory 39: 439-467.

Goodin, Robert E. 1988. What is so Special about Our Fellow Countrymen? Ethics 98: 663-686.

Griffin, James. 2008. On Human Rights. Oxford: Oxford University Press.

Habermas, Jürgen. 1996. Die Einbeziehung des Anderen. Frankfurt a. M.: Suhrkamp Verlag.

Höffe, Otfried. 2009. Anthropologie und Menschenrechte. Zum politischen Projekt der Moderne. In Politische Anthropologie. Geschichte - Gegenwart-Möglichkeiten, Hrsg. Dirk Jörke und Bernd Ladwig, 231-244. Baden-Baden: Nomos Verlag.

Ignatieff, Michael. 2001. Human Rights as Politics and as Idolatry. Princeton, N. J.: Princeton University Press. 
James, Susan. 2003. Rights as Enforceable Claims. Proceedings of the Aristotelian Society 103: 133-147.

Kant, Immanuel. [1797] 1968: Die Metaphysik der Sitten. Werkausgabe Band VIII. Hrsg. Wilhelm Weischedel. Frankfurt a. M.: Suhrkamp Verlag.

Kant, Immanuel. [1798] 1991. Der Streit der Fakultäten. In ders., Schriften zur Anthropologie, Geschichtsphilosophie, Politik und Pädagogik 1. Werkausgabe Band XI. Hrsg. Wilhelm Weischedel. Frankfurt a. M.: Suhrkamp Verlag.

Kreide, Regina. 2007. Weltarmut und die Verpflichtungen kollektiver Akteure. In Weltarmut und Ethik, Hrsg. Barbara Bleisch und Peter Schaber, 267-295. Paderborn: Mentis Verlag.

Kymlicka, Will. 1996. Two Models of Pluralism and Tolerance. In Toleration. An Elusive Virtue, Hrsg. David Heyd, 81-105. Princeton, N. J.: Princeton University Press.

Ladwig, Bernd. 2011. Gerechtigkeitstheorien zur Einführung. Hamburg: Junius Verlag.

Ladwig, Bernd. 2013. Menschenrechte als Grundnormen der politischen Moral. In Menschenrechte und Demokratie. Georg Lohmann zum 65. Geburtstag, Hrsg. Falk Bornmüller, Thomas Hoffmann und Arnd Pollmann, 43-66. Freiburg/München: Verlag Karl Alber.

Ladwig, Bernd, und Beate Rudolf. 2011. International Legal and Moral Standards of Good Governance in Fragile States. In Governance Without a State. Policies and Politics in Areas of Limited Statehood, Hrsg. Thomas Risse, 199-231. New York: Columbia University Press.

Lafont, Cristina. 2011. Global Governance and Human Rights. Spinoza Lectures. Assen: Koninklijke Van Gorcum.

Lohmann, Georg. 1998. Menschenrechte zwischen Moral und Recht. In Philosophie der Menschenrechte, Hrsg. Stefan Gosepath und Georg Lohmann, 62-95. Frankfurt a. M.: Suhrkamp Verlag.

Luhmann, Niklas. 1997. Die Gesellschaft der Gesellschaft. Frankfurt a. M.: Suhrkamp Verlag.

Margalit, Avishai. 1997. Politik der Würde. Über Achtung und Verachtung. Berlin: Alexander Fest Verlag.

Menke, Christoph, und Arnd Pollmann. 2007. Philosophie der Menschenrechte zur Einführung. Hamburg: Junius Verlag.

Menke, Christoph, und Francesca Raimondi (Hrsg.) 2011. Die Revolution der Menschenrechte. Grundlegende Texte zu einem neuen Begriff des Politischen. Frankfurt a. M.: Suhrkamp Verlag.

Mieth, Corinna. 2012. Positive Pflichten. Über das Verhältnis von Hilfe und Gerechtigkeit in Bezug auf das Weltarmutsproblem. Berlin: De Gruyter.

Miller, David. 2007. National Responsibility and Global Justice. Oxford: Oxford University Press.

Murphy, Liam. 2000. Moral Demands in Nonideal Theory. Oxford: Oxford University Press.

Neuhäuser, Christian. 2011. Unternehmen als moralische Akteure. Frankfurt a. M.: Suhrkamp Verlag.

Nickel, James. 2007. Making Sense of Human Rights. Second Edition. Oxford: Blackwell Publishing. 
O’Neill, Onora. 1996. Towards Virtue and Justice. A Constructive Account of Practical Reasoning. Cambridge: Cambridge University Press.

Pogge, Thomas. 2002. World Poverty and Human Rights: Cosmopolitan Responsibilities and Reforms. Cambridge: Polity Press.

Rawls, John. 1975. Eine Theorie der Gerechtigkeit. Frankfurt a. M.: Suhrkamp Verlag.

Rawls, John. 2002. Das Recht der Völker. Berlin: De Gruyter.

Raz, Joseph. 2010. Human Rights Without Foundations. In The Philosophy of International Law, Hrsg. Samantha Besson und John Tasioulas, 321-338. Oxford: Oxford University Press.

Risse, Thomas (Hrsg.). 2011. Governance Without a State. Policies and Politics in Areas of Limited Statehood. New York: Columbia University Press.

Risse, Thomas, Stephen C. Ropp, und Kathryn Sikkink (Hrsg.). 2013. The Persistent Power of Human Rights. From Commitment to Compliance. Cambridge: Cambridge University Press.

Schmelzle, Cord. 2012. Politische Legitimität und zerfallene Staatlichkeit. Dissertation an der Freien Universität Berlin. Manuskript.

Sen, Amartya. 2010. Die Idee der Gerechtigkeit. München: Verlag C. H. Beck.

Shue, Henry. 1980. Basic Rights. Subsistence, Affluence, and U.S. Foreign Policy. Princeton, N. J.: Princeton University Press.

Shue, Henry. 1988. Mediating Duties. Ethics 98: 687-704.

Singer, Peter. 1972. Famine, Affluence, and Morality. In Philosophy and Public Affairs 3: 229-243.

Strawson, Peter. 1962. Freedom and Resentment. Proceedings of the British Academy 48: $1-25$.

Tasioulas, John. 2009. Are Human Rights Essentially Triggers for Intervention? Philosophy Compass 6: 938-950. doi: 10.1111/j.1747-9991.2009.00246.x.

Tasioulas, John. 2010. Taking Rights out of Human Rights. Ethics 120: 647-678.

Tuck, Richard. 1977. Natural Rights Theories. Their Origin and Development. Cambridge: Cambridge University Press.

Tugendhat, Ernst. 1993. Vorlesungen über Ethik. Frankfurt a. M.: Suhrkamp Verlag.

Valentini, Laura 2011. In What Sense are Human Rights Political? A Preliminary Exploration. Political Studies 60: 180-194.

Walzer, Michael. 1996. Lokale Kritik - globale Standards. Zwei Formen moralischer Auseinandersetzung. Hamburg: Rotbuch Verlag.

Wellmer, Albrecht. 1999. Hannah Arendt über die Revolution. In Recht auf Menschenrechte. Menschenrechte, Demokratie und internationale Politik, Hrsg. Hauke Brunkhorst, Wolfgang R. Köhler und Matthias Lutz-Bachmann, 125-156. Frankfurt a. M.: Suhrkamp Verlag.

\section{Autorenangaben}

Prof. Dr. Bernd Ladwig,

Freie Universität Berlin, Otto-Suhr-Institut für Politikwissenschaft,

Ihnestraße 22,

14195 Berlin,

ladwig@zedat.fu-berlin.de 